\title{
Exact Multi-soliton Solution for the (3+1)-D Jimbo-Miwa Equation
}

\author{
Xichao Deng \\ Applied Technology College, Southwest University of Science and Technology \\ Mianyang 621010, China \\ Zhenhui Xu (Corresponding author) \\ Applied Technology College, Southwest University of Science and Technology \\ Mianyang 621010, China
}

Tel: 86-134-5830-2193 E-mail: xuzhenhui19@163.com

Received: August 4, 2011 Accepted: August 29, 2011 Published: November 1, 2011

doi:10.5539/jmr.v3n4p89 URL: http://dx.doi.org/10.5539/jmr.v3n4p89

This work was supported by Chinese Natural Science Foundation Grant No.11061028,10971169. Sichuan Educational science Foundation Grant No.09zc008.

\begin{abstract}
In this paper, by using bilinear form and extended three-wave type of ansätz approach, we obtain new multi-soliton solutions of the (3+1)-dimensional Jimbo-Miwa equation, including the periodic breather-type of kink three-soliton solutions, the cross-kink four-soliton solutions, the doubly periodic breather-type of soliton solutions and the doubly periodic breather-type of cross-kink two-soliton solutions. It is shown that the generalized three-wave method, with the help of symbolic computation, provides an effective and powerful mathematical tool for solving high dimensional nonlinear evolution equations in mathematical physics.
\end{abstract}

Keywords: Jimbo-Miwa equation, Extended three-wave method, Multi-soliton solution

\section{Introduction}

It is well known that many important phenomena in physics and other fields are described by nonlinear partial differential equations. As mathematical models of these phenomena, the investigation of exact solutions is important in mathematical physics. Many methods are available to look for exact solutions of nonlinear evolution equations, such as the inverse scattering method, the Lie group method, the mapping method, Exp-function method, ansätz technique, three-wave tape of ansätz approach and so on (Ma and Fan(2011),Liu et al.(2005),Dai et al.(2005)). In this paper, we consider the following Jimbo-Miwa equation:

$$
u_{x x y}+3 u_{x} u_{x y}+3 u_{x x} u_{y}+2 u_{y t}-3 u_{x z}=0
$$

By means of the two-soliton method and bilinear methods, the the two-soliton solutions, three-wave solutions of the Jimbo-Miwa were found as well as (Dai et al.(2007),Li et al.(2011)).

In this paper, we discuss further the $(3+1)$-dimensional Jimbo-Miwa equation, by using bilinear form and extend threewave type of ansätz approach, respectively (Xu and Xian (2010), Chow(2002), Xu and Liu (2010)), Some new multisoliton solutions are obtained.

\section{The multi-soliton solutions}

We assume

$$
u=2(\ln f)_{x}
$$

where $f=f(x, y, z, t)$ is unknown real function. Substituting Eq.(2) into Eq.(1), we can reduce Eq.(1) into the following Hirota bilinear equation

$$
\left(D_{x}^{3} D_{y}+2 D_{y} D_{t}-3 D_{x} D_{z}\right) f \cdot f=0
$$

where the Hirota bilinear operator $\mathrm{D}$ is defined by $(\mathrm{n}, \mathrm{m} \geq 0)$

$$
D_{x}^{m} D_{t}^{n} f(x, t) \cdot g(x, t)=\left.\left(\frac{\partial}{\partial x}-\frac{\partial}{\partial x^{\prime}}\right)^{m}\left(\frac{\partial}{\partial t}-\frac{\partial}{\partial t^{\prime}}\right)^{n}\left[f(x, t) g\left(x^{\prime}, t^{\prime}\right)\right]\right|_{x^{\prime}=x, t^{\prime}=t}
$$


Now we suppose the solution of Eq.(3) as

$$
f=e^{-\xi}+\delta_{1} \cos (\eta)+\delta_{2} \sinh (\gamma)+\delta_{3} \cosh (\theta)+\delta_{4} e^{\xi}
$$

where $\xi=a_{1} x+b_{1} y+c_{1} z+d_{1} t, \eta=a_{2} x+b_{2} y+c_{2} z+d_{2} t, \gamma=a_{3} x+b_{3} y+c_{3} z+d_{3} t, \theta=a_{4} x+b_{4} y+c_{4} z+d_{4} t$, and $a_{i}, b_{i}, c_{i}, d_{i}(i=1,2,3,4)$ are some constants to be determined later. Substituting Eq.(5) into Eq.(3) and equating all the coefficients of different powers of $e^{\xi}, e^{-\xi}, \sin (\eta), \cos (\eta), \sinh (\gamma), \cosh (\gamma), \sinh (\theta), \cosh (\theta)$ and constant term to zero, we can obtain a set of algebraic equations for $a_{i}, b_{i}, c_{i}, d_{i}, \delta_{j}(i=1,2,3,4 ; j=1,2,3,4)$. Solving the system with the aid of Maple, we get the following results:

Case(I):

$$
\left\{\begin{array}{l}
a_{1}=0, a_{3}=0, b_{2}=0, b_{4}=0, c_{2}=0, c_{3}=\frac{b_{3} c_{1}}{b_{1}}, c_{4}=0, d_{1}=0, d_{3}=0 \\
d_{2}=\frac{a_{2}\left(a_{2}^{2} b_{1}-3 c_{1}\right)}{2 b_{1}}, d_{4}=-\frac{a_{4}\left(3 c_{1}+a_{4}^{2} b_{1}\right)}{2 b_{1}}, \delta_{1}=\delta_{1}, \delta_{2}=\delta_{2}, \delta_{3}=\delta_{3}, \delta_{4}=\delta_{4}
\end{array}\right.
$$

where $a_{2}, a_{4}, b_{1}, b_{3}, c_{1}, \delta_{1}, \delta_{2}, \delta_{3}, \delta_{4}$ are some free real constants. Substituting Eq.(6) into Eq.(5) and taking $\delta_{4}>0$, we have

$$
f_{1}=2 \sqrt{\delta_{4}} \cosh \left(b_{1} y+c_{1} z+\frac{1}{2} \ln \left(\delta_{4}\right)+\delta_{1} \cos \left(a_{2} x+K_{1} t\right)+\delta_{2} \sinh \left(b_{3} y+L_{1} z\right)+\delta_{3} \cosh \left(a_{4}-H_{1} t\right)\right.
$$

where $K_{1}=\frac{a_{2}\left(a_{2}^{2} b_{1}-3 c_{1}\right)}{2 b_{1}}, L_{1}=\frac{b_{3} c_{1}}{b_{1}}, H_{1}=\frac{a_{4}\left(3 c_{1}+a_{4}^{2} b_{1}\right)}{2 b_{1}}$. Substituting Eq.(7) into Eq. (2) yields the periodic breather-type of kink three-soliton solutions for Jimbo-Miwa equation as follows:

$$
u_{1}=-\frac{2\left[a_{2} \delta_{1} \sin \left(a_{2} x+K_{1} t\right)-a_{4} \delta_{3} \sinh \left(a_{4} x-H_{1} t\right)\right]}{2 \sqrt{\delta_{4}} \cosh \left(b_{1} y+c_{1} z+\frac{1}{2} \ln \left(\delta_{4}\right)+\delta_{1} \cos \left(a_{2} x+K_{1} t\right)+\delta_{2} \sinh \left(b_{3} y+L_{1} z\right)+\delta_{3} \cosh \left(a_{4}-H_{1} t\right) .\right.}
$$

The figure of $u_{1}$ as $\delta_{1}=\frac{1}{2}, \delta_{2}=\frac{1}{4}, t=0$ refer Fig. (a).

If taking $a_{2}=i A_{2}$ in Eq.(7), then we have

$$
f_{2}=2 \sqrt{\delta_{4}} \cosh \left(b_{1} y+c_{1} z+\frac{1}{2} \ln \left(\delta_{4}\right)+\delta_{1} \cosh \left(A_{2} x-K_{2} t\right)+\delta_{2} \sinh \left(b_{3} y+L_{1} z\right)+\delta_{3} \cosh \left(a_{4}-H_{1} t\right)\right.
$$

where $\delta_{4}>0, K_{2}=\frac{A_{2}\left(A_{2}^{2} b_{1}+3 c_{1}\right)}{2 b_{1}}$. Substituting Eq.(9) into Eq.(2) yields the cross-kink four-soliton solutions of Jimbo-Miwa equation as follows:

$$
u_{2}=\frac{2\left[A_{2} \delta_{1} \sinh \left(A_{2} x-K_{2} t\right)+a_{4} \delta_{3} \sinh \left(a_{4} x-H_{1} t\right)\right]}{2 \sqrt{\delta_{4}} \cosh \left(b_{1} y+c_{1} z+\frac{1}{2} \ln \left(\delta_{4}\right)+\delta_{1} \cosh \left(A_{2} x-K_{2} t\right)+\delta_{2} \sinh \left(b_{3} y+L_{1} z\right)+\delta_{3} \cosh \left(a_{4}-H_{1} t\right) .\right.}
$$

The figure of $u_{2}$ as $\delta_{1}=\frac{1}{2}, \delta_{2}=\frac{1}{5}, t=1$ refer Fig. 2 .

If taking $a_{4}=i A_{4}$ in Eq.(7), then we have

$$
f_{3}=2 \sqrt{\delta_{4}} \cosh \left(b_{1} y+c_{1} z+\frac{1}{2} \ln \left(\delta_{4}\right)+\delta_{1} \cos \left(a_{2} x+K_{1} t\right)+\delta_{2} \sinh \left(b_{3} y+L_{1} z\right)+\delta_{3} \cos \left(A_{4}+H_{2} t\right)\right.
$$

where $\delta_{4}>0, H_{2}=\frac{A_{4}\left(A_{4}^{2} b_{1}-3 c_{1}\right)}{2 b_{1}}$. Substituting Eq.(11) into Eq.(2) yields the doubly periodic breather-type of soliton solutions for Jimbo-Miwa equation as follows:

$$
u_{3}=-\frac{2\left[a_{2} \delta_{1} \sin \left(a_{2} x+K_{1} t\right)+A_{4} \delta_{3} \sin \left(A_{4} x+H_{2} t\right)\right]}{2 \sqrt{\delta_{4}} \cosh \left(b_{1} y+c_{1} z+\frac{1}{2} \ln \left(\delta_{4}\right)+\delta_{1} \cos \left(a_{2} x+K_{1} t\right)+\delta_{2} \sinh \left(b_{3} y+L_{1} z\right)+\delta_{3} \cos \left(A_{4}+H_{2} t\right) .\right.}
$$

The figure of $u_{3}$ as $\delta_{1}=\frac{1}{5}, \delta_{2}=\frac{1}{2}, t=0$ refer Fig. 3.

If taking $a_{4}=i A_{4}, b_{3}=i B_{3}, \delta_{2}=i Q_{2}$ in Eq.(7), then we have

$$
f_{4}=2 \sqrt{\delta_{4}} \cosh \left(b_{1} y+c_{1} z+\frac{1}{2} \ln \left(\delta_{4}\right)+\delta_{1} \cos \left(a_{2} x+K_{1} t\right)-Q_{2} \sin \left(B_{3} y+L_{2} z\right)+\delta_{3} \cos \left(A_{4}+H_{2} t\right)\right.
$$

where $A_{4}, B_{3}, Q_{2}$ are some free real constants, $L_{2}=\frac{B_{3} c_{1}}{b_{2}}$ and $\delta_{3}>0$. Substituting Eq.(13) into Eq.(2) yields the doubly periodic breather-type of soliton solutions for Jimbo-Miwa equation as follows:

$$
u_{4}=-\frac{2\left[a_{2} \delta_{1} \sin \left(a_{2} x+K_{1} t\right)+A_{4} \delta_{3} \sin \left(A_{4} x+H_{2} t\right)\right]}{2 \sqrt{\delta_{4}} \cosh \left(b_{1} y+c_{1} z+\frac{1}{2} \ln \left(\delta_{4}\right)+\delta_{1} \cos \left(a_{2} x+K_{1} t\right)-Q_{2} \sin \left(B_{3} y+L_{2} z\right)+\delta_{3} \cos \left(A_{4}+H_{2} t\right) .\right.}
$$


The figure of $u_{4}$ as $\delta_{1}=1, \delta_{2}=\frac{1}{3}, t=0$ refer Fig. 4 .

Case(II):

$$
\left\{\begin{array}{l}
a_{1}=0, a_{2}=\frac{\sqrt{4-a_{4}^{4}}}{a_{4}}, a_{3}=\frac{2 i}{a_{4}}, b_{1}=1, b_{2}=0, b_{3}=\frac{i a_{4}^{2}}{\sqrt{4-a_{4}^{4}}}, b_{4}=-\frac{2}{\sqrt{4-a_{4}^{4}}}, c_{2}=1 \\
c_{3}=\frac{i\left(a_{4}^{2} c_{1}+2\right)}{\sqrt{4-a_{4}^{4}}}, c_{4}=\frac{a_{4}^{2}-2 c_{1}}{\sqrt{4-a_{4}^{4}}}, d_{1}=\frac{3 \sqrt{4-a_{4}^{4}}}{2 a_{4}}, d_{2}=-\frac{\left(3 a_{4}^{2} c_{1}+a_{4}^{4}-4\right) \sqrt{4-a_{4}^{4}}}{2 a_{4}^{3}} \\
d_{3}=-\frac{i\left(3 a_{4}^{4}+6 a_{4}^{2} c_{1}-8\right)}{2 a_{4}^{3}}, d_{4}=-\frac{\left.3 a_{4}^{2} c_{1}+a_{4}^{4}-6\right)}{2 a_{4}}, \delta_{4}=\frac{a_{4}^{4}\left(\delta_{2}^{2}+\delta_{3}^{2}-\delta_{1}^{2}\right)+4\left(\delta_{1}^{2}+\delta_{2}^{2}+\delta_{3}^{2}\right)}{4\left(4-a_{4}^{4}\right)}
\end{array}\right.
$$

where $a_{4}, c_{1}, \delta_{1}, \delta_{2}, \delta_{3}$ are some free real constants. Substituting Eq.(15) into Eq.(5) and taking $M>0$, we have

$$
f_{5}=2 \sqrt{M} \cosh \left(\xi+\frac{1}{2} \ln (M)\right)+\delta_{1} \cos (\eta)+\delta_{2} \sin (\gamma)+\delta_{3} \cosh (\theta)
$$

when $M>0$.where $\xi=a_{1} x+y+c_{1} z+\frac{3 \sqrt{4-a_{4}^{4}}}{2 a_{4}} t, \eta=\frac{\sqrt{4-a_{4}^{4}}}{a_{4}} x+z-\frac{\left(3 a_{4}^{2} c_{1}+a_{4}^{4}-4\right) \sqrt{4-a_{4}^{4}}}{2 a_{4}^{3}} t, \gamma=\frac{2}{a_{4}} x+\frac{a_{4}^{2}}{\sqrt{4-a_{4}^{4}}} y+\frac{\left(a_{4}^{2} c_{1}+2\right)}{\sqrt{4-a_{4}^{4}}} z-$ $\frac{\left(3 a_{4}^{4}+6 a_{4}^{2} c_{1}-8\right)}{2 a_{4}^{3}} t, \theta=a_{4} x-\frac{2}{\sqrt{4-a_{4}^{4}}} y+\frac{a_{4}^{2}-2 c_{1}}{\sqrt{4-a_{4}^{4}}} z-\frac{\left.3 a_{4}^{2} c_{1}+a_{4}^{4}-6\right)}{2 a_{4}} t, M=\frac{a_{4}^{4}\left(\delta_{2}^{2}+\delta_{3}^{2}-\delta_{1}^{2}\right)+4\left(\delta_{1}^{2}+\delta_{2}^{2}+\delta_{3}^{2}\right)}{4\left(4-a_{4}^{4}\right)}$. Substituting Eq.(16) into Eq.(2), we obtain the doubly periodic breather-type of cross-kink two-soliton solutions for Jimbo-Miwa equation as follows:

$$
u_{5}=\frac{2\left[2 a_{1} \sqrt{M} \sinh \left(\xi+\frac{1}{2} \ln (M)\right)+\frac{\delta_{1} \sqrt{4-a_{4}^{4}}}{a_{4}} \sin (\eta)-\frac{2 \delta_{2}}{a_{4}} \cos (\gamma)-a_{4} \delta_{3} \sinh (\theta)\right]}{2 \sqrt{M} \cosh \left(\xi+\frac{1}{2} \ln (M)\right)+\delta_{1} \cos (\eta)+\delta_{2} \sin (\gamma)+\delta_{3} \cosh (\theta)}
$$

\section{Conclusion}

By using bilinear form and extended three-wave type of ansätz approach, we discuss further the $(3+1)$-dimensional Jimbo-Miwa equation and find some new multi-soliton solutions. The results show that the extended three-wave tape of ansätz approach may provide us with a straightforward and effective mathematical tool for seeking multi-wave solutions of higher dimensional nonlinear evolution equations.

\section{References}

Chow, K.W. (2002). A class of doubly periodic waves for nonlinear evolution equations. Wave Motion, 35,71-90. http://dx.doi.org/10.1016/S0165-2125(01)00078-6

Dai, Z. D., Huang, J., Jiang, M. R. \& Wang, S. H. (2005). Homoclinic orbits and periodic solitons for Boussinesq equation with even constraint. Chaos Soliton and Fractals, 26,1189-1194. http://dx.doi.org/10.1016/j.chaos.2005.02.025

Dai, Z. D., Li, Z. T., Liu, Z. J. \& Li, D. L. (2007). Exact cross kink-wave solutions and resonance for the JimboCMiwa equation. Physica A, 384, 285C290.

Li, Z. T., Dai, Z. D. \& Liu, J. (2011). Exact three-wave solutions for the $(3+1)$-dimensional JimboCMiwa equation. Computers and Mathematics with Applications, 61,2062C2066.

Liu, X. Q., Chen, H. L. \& Lv, Y. Q. (2005). Explicit solutions of the generalized KdV equation with higher order nonlinearity. Appl. Math. Comput., 171,315-319. http://dx.doi.org/10.1016/j.amc.2005.01.053

Ma, W. X. \& Fan, E. G. (2011). Linear superposition principle applying to Hirota bilinear equations. Computers and Mathematics with Applications, 61, 950-959. http://dx.doi.org/10.1016/j.camwa.2010.12.043

Xu, Z. H. \& Liu, X. Q. (2010). Explicit Peaked Wave Solution to the Generalized Camassa-Holm Equation. Acta Mathematicae Applicatae Sinica, 26(2),277-282. http://dx.doi.org/10.1007/s10255-008-8116-8

Xu, Z. H. \& Xian, D. Q. (2010). New periodic solitary-wave solutions for the Benjiamin Ono equation. Applied Mathematics and Computation, 215, 4439-4442. http://dx.doi.org/10.1016/j.amc.2009.11.009 

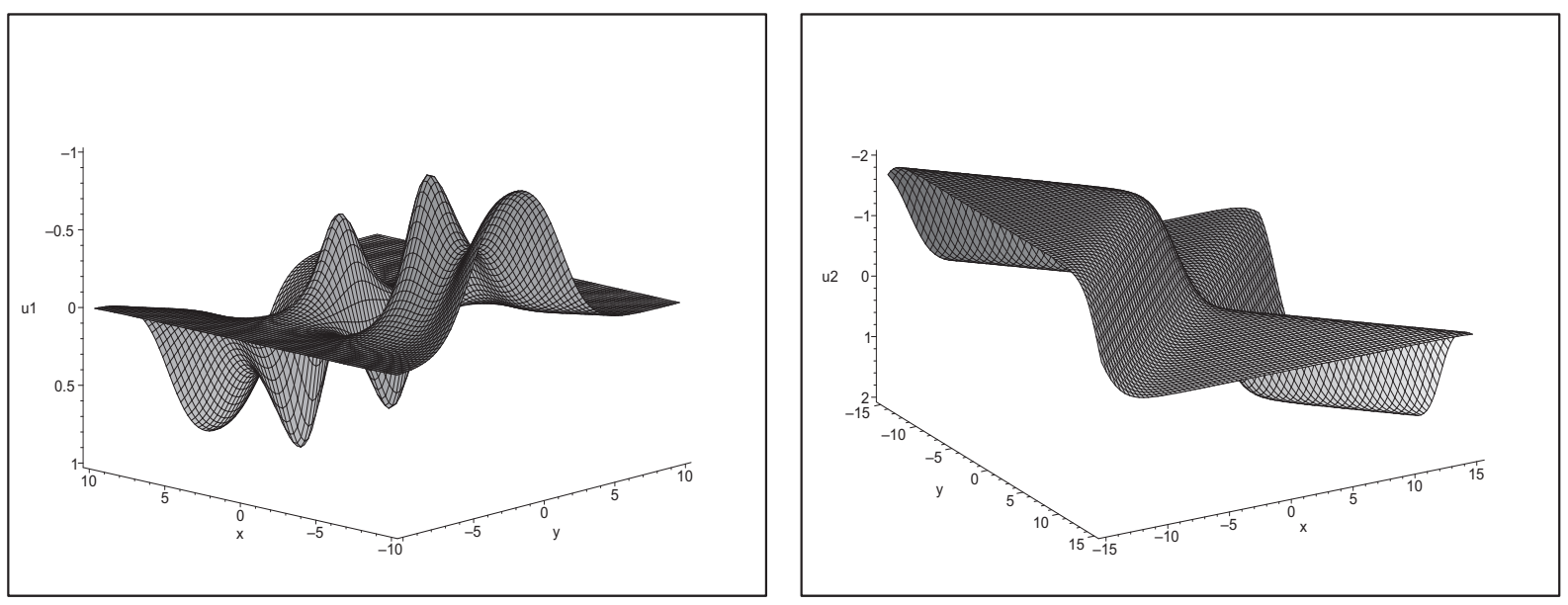

Figure 1. The figure of $u_{1}$ as $\delta_{1}=\frac{1}{2}, \delta_{2}=\frac{1}{4}, t=0$

Figure 2. The figure of $u_{2}$ as $\delta_{1}=\frac{1}{2}, \delta_{2}=\frac{1}{5}, t=1$
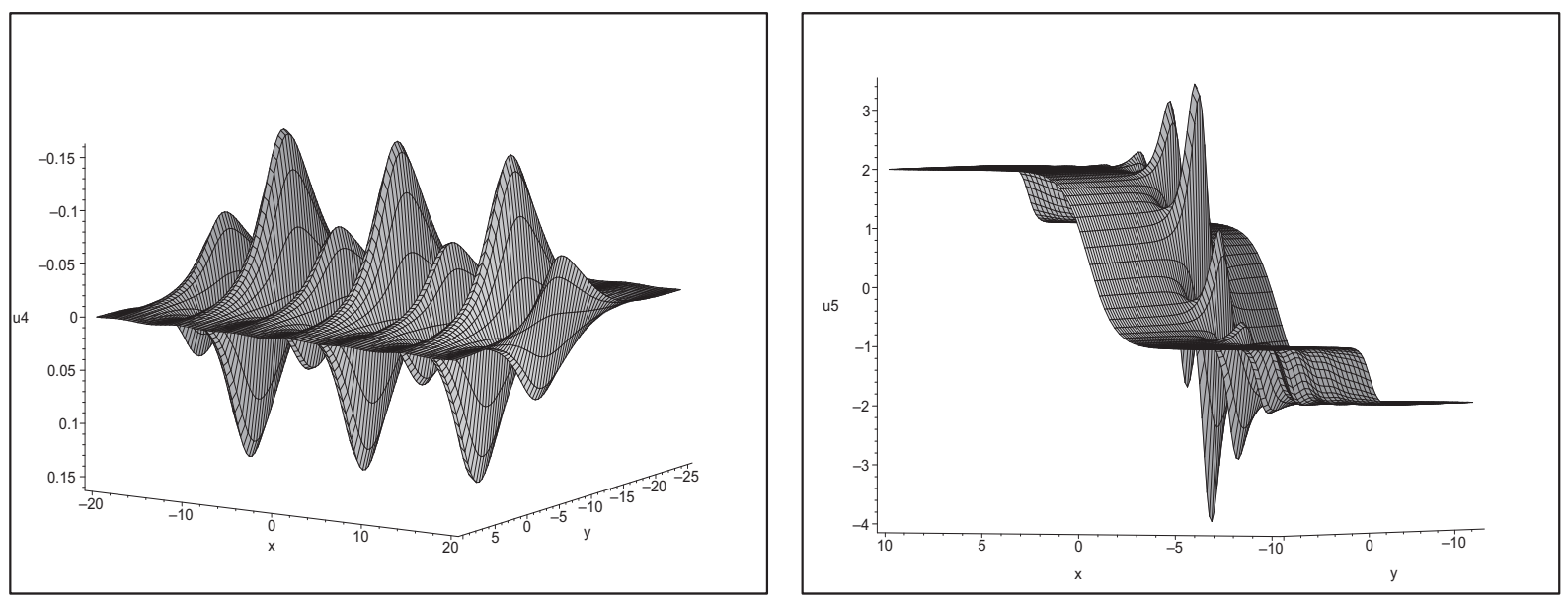

Figiure 3. The figure of $u_{3}$ as $\delta_{1}=\frac{1}{5}, \delta_{2}=\frac{1}{2}, t=0$

Figure 4.The figure of $u_{4}$ as $\delta_{1}=1, \delta_{2}=\frac{1}{3}, t=0$ 